\title{
Reference Intervals in Malaysia: A Performance Evaluation and Comparison of Haematological Parameters between Sysmex $\mathrm{XE}-5000$ and $\mathrm{XN}-3000$
}

\section{Siew Lian CHONG ${ }^{1 *}$, Asral Wirda AHMAD ASNAWI ${ }^{1,2}$, Kian Boon LAW ${ }^{3}$, Roszymah HAMZAH ${ }^{1}$ and Sen MUI TAN ${ }^{1}$}

${ }^{1}$ Department of Haematology, Hospital Ampang, Selangor, Malaysia

${ }^{2}$ Faculty of Medicine and Health Sciences, Universiti Sains Islam Malaysia, Negeri Sembilan, Malaysia

${ }^{3}$ Institute for Clinical Research, National Institutes of Health (NIH), Selangor, Malaysia

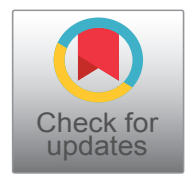

*Corresponding author: Siew Lian CHONG, Department of Haematology, Hospital Ampang, Jalan Mewah Utara, Pandan Mewah, 68000 Ampang, Selangor, Malaysia; Tel: +60342896000

\begin{abstract}
The Sysmex $\mathrm{XN}-3000$ is a new automated haematology analyser designed to improve the accuracy of cell counts and the specificity of the flagging events of unusual parameters. By comparing the previous full blood count (FBC) reference intervals in Malaysia for Sysmex XE5000 , we determined a reference interval for all parameters measured by the Sysmex XN-3000 for the Malaysian population. Through the voluntary recruitment of 397 adults ages $18-45$ years, both genders, and the three main ethnic groups, FBC was performed on the two analysers. Qualified healthy participants were screened using a health questionnaire. This was followed by reference intervals, probability distribution measurements, and dispersion with point estimate determination. Complete data were available in 390 subjects comprising 222 females and 168 males, which were included in the reference interval calculation. Parameters such as haemoglobin, red blood cell count, platelet count including immature platelet fraction (IPF) showed significant differences in Malaysians. XN-3000 showed excellent precision and linearity results. Within- and between-run precisions were met for all parameters tested, except for IPF. For all parameters tested, $\leq 0.5 \%$ carry-over was seen. An acceptable correlation with both XN-3000 and XE-5000 was achieved in comparison studies performed. $\mathrm{XN}-3000$ showed good analytical performance and could provide a solution for laboratories with medium-to-high workloads and evolving clinical needs. Local guidelines are required for the establishment of reference intervals.
\end{abstract}

\section{Keywords}

Sysmex, Laboratory practice, Statistics, General hematology, XN-3000

\section{Introduction}

The use of specific instruments and novel chemical agents dictate the applicability of haematological reference intervals. Normal parameter determination is subject to demographic and inherent factors as well as many pre-analytical variables dictating that native laboratory limits need to be determined for every reported parameter on any given analyse [1]. Multiple factors can influence physiological values in a normal healthy population, thus creating a mandatory requirement for the development of a set of acceptable reference values for a particular population before applying the results generated into any clinical situation or pathological condition [2]. In clinical practice, effective translation and differentiation between a state of well-being and disease essentially require that precise reference ranges correctly represent the people subjected to the analysis. This may cut or deflect unnecessary follow-up examinations of patients while reducing medical costs. Reference values for various laboratory tests are one of the most important elements in clinical practice. These values can be adopted by using values given by manufacturer or reference laboratory, using values obtained in relevant published articles, using previous values in own laboratory, or established locally. It is noteworthy that individual laboratories rarely verify the adopted value using samples from

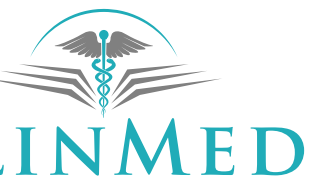

INTERNATIONAL LIBRARY
Citation: CHONG SL, ASNAWI AWA, LAW KB, HAMZAH R, TAN SM (2021) Reference Intervals in Malaysia: A Performance Evaluation and Comparison of Haematological Parameters between Sysmex XE-5000 and XN-3000. Int J Blood Res Disord 8:072. doi.org/10.23937/2469-5696/1410072

Accepted: December 21, 2021: Published: December 23, 2021

Copyright: @ 2021 CHONG SL, et al. This is an open-access article distributed under the terms of the Creative Commons Attribution License, which permits unrestricted use, distribution, and reproduction in any medium, provided the original author and source are credited. 
human subjects, nor establish their own reference value.

Reference values describing the dispersion of test values in healthy individuals and are typically reported as population-based reference interval (RI) comprising $95 \%$ of the healthy population $[3,4]$. The standard for the production of human populationbased RI was commissioned by the international federation of clinical chemistry (IFCC) and Laboratory Medicine in 1970 [5]. The expert panel on the theory of reference values (EPTRV) authored a 6-part series on the production of reference values, which was adopted by several professional organizations including the clinical and laboratory standards institute (CLSI) [2,59]. Subsequently, the proposal of adding alternative statistical methods for the identification of outliers, analysis of reference values, and determination of the need for partitioning was made. Reference values are obtained from a healthy reference group would help clinicians interpret the test results of their patients that play into the decision-making process in charting the next course of action in management.

The latest Malaysian population-based reference values for full blood count (FBC) were established in 2004 by Ambayya, et al., using Sysmex XE 5000 (Sysmex, Kobe, Japan) and Unicel DxH 800 (Beckman Coulter, USA) haemato-analysers, following the international council for standardization in haematology (ICSH) guidelines. In this large-scale study, many ancillary investigations were performed to demonstrate its correlation with the haematological parameters [10]. These included iron studies, haemoglobinopathy screening by capillary electrophoresis, and markers of inflammation. Since then, the Malaysian populationbased FBC reference values have been adopted by local hospital laboratories across the country in reporting FBC and by physicians involved in the clinical practice. The Sysmex XN modular system is a new generation analyser using different principles, channels, and reagents from the Sysmex XE model. Its performance on adult peripheral blood samples has been reported in a few studies [11-13]. The commencement of a new haematology analyser, Sysmex XN-3000 at the makmal rujukan klinikal hematologi (MRKH) of the Department of Hematology, Hospital Ampang introduced some advanced test parameters such as immature granulocytes (IG), reticulocyte hemoglobin (RET-He), immature reticulocyte (IRF), immature platelet fraction (IPF) and fluorescent platelet count (PLT-F), that could be useful into daily hematological service. To verify that all current and advanced test parameters of the new haematology analyser are in agreement with the Malaysian population-based reference values, a study of verification, re-verification, and establishment of reference values using samples from healthy reference individuals was performed. This study aimed to assess the agreement between the Malaysian population- based FBC reference intervals and the new Sysmex hematology analyser, model XN-3000 with emphasis on advanced clinical parameters and transference of ranges from XE-5000.

\section{Materials and Methods}

\section{Selection of a reference sample group}

This study was approved by the Medical Research and Ethics Committee of the Ministry of Health, Malaysia (NMRR-17-606-35642). Blood samples were collected from healthy male and female individuals, aged between 18 and 45 years. This is under the international council for standardization in haematology (ICSH) guidelines with regards to the sampling method for determining normal values of haematology [14]. A priori approach was applied for all healthy donors. Health assessment and blood specimen collection were executed almost at the same time after obtaining a written consent by the participant. Common disorders such as haemoglobinopathy and iron deficiency anaemia were excluded based on their FBC on the XE-5000 established reference values that had been obtained in a normal healthy Malaysia population. Any volunteers with abnormal results were referred to the local health practitioner for assessment. Reliable transference or the adoption of new reference values was performed against the previously established standards that had been done previously by our laboratory. Therefore, the establishment of reference intervals required minimal sample size as per CLSI guidelines. Thus, a total of 168 males and 222 females were evaluated and included in the study. Some volunteers were excluded from this study. This included volunteers with a history of smoking tobacco and female volunteers having menses at the time of sampling.

\section{Blood sampling and processing of samples}

EDTA bottles were used for blood collection for analysis of FBC by the new model, Sysmex XN-3000 against the established model, Sysmex XE-5000 (Sysmex, Kobe, Japan) analyser. Full blood counts were analysed within 1 hour of the collection of blood samples. An adopted reference interval was first verified using samples obtained from 20 reference individuals. If $\geq$ $90 \%$ of samples ( $\geq 18$ samples) are within the reference intervals, the transference is verified. If $<90 \%$ of samples are within the reference intervals, re-verification will be carried out using 20 additional samples obtained from another 20 reference individuals. If $\geq 90 \%$ of samples ( $\geq 18$ samples) are within the reference intervals, the transference is verified.

\section{Statistical analysis}

The mean/median and ranges of all parameters on XN-3000 and XE-5000 were evaluated. Normality of the test parameters were tested using Kolmogorov-Smirnov Z-test. Probability distribution was expressed as mean 
or median where appropriate. Calculation of dispersion includes standard deviation (SD), range, and interquartile range (IQR). Other point estimates for the test parameters were calculated in total and according to gender (values at $2.5^{\text {th }}, 25.0^{\text {th }}, 75.0^{\text {th }}$, and $97.5^{\text {th }}$ ). A non- parametrical percentile method (2.5-97.5 percentiles) was used to calculate reference intervals with an estimation of the lower and upper reference limits according to the CLSI guidance document (C28-A3) [15]. For each reference limit, 90\% confidence intervals (CI)

Table 1: Reference ranges for parameters reported on Sysmex XN-3000 (Male, $N=168$ ).

\begin{tabular}{|c|c|c|c|c|c|c|c|c|c|}
\hline \multirow[b]{2}{*}{ Parameters } & \multirow[b]{2}{*}{$\mathbf{n}$} & \multicolumn{8}{|c|}{ Shapiro-Wilk test for normality } \\
\hline & & p-value & Variance & Mean & SD & $\begin{array}{l}\text { Range } \\
\text { (mean } \pm 2 S D \text { ) }\end{array}$ & Median & IQR & $\begin{array}{l}\text { Range } \\
\left(2.5^{\text {th }}-97.5^{\text {th }}\right. \\
\text { percentiles })\end{array}$ \\
\hline $\mathrm{WBC}^{\dagger}$ & 168 & 0.102 & 2.11 & 7.24 & 1.45 & $4.34-10.15$ & & & \\
\hline $\mathrm{RBC}^{\ddagger}$ & 168 & 0.111 & 0.12 & 5.34 & 0.35 & $4.64-6.03$ & & & \\
\hline HGB (g/dl) & 168 & 0.791 & 0.82 & 15.50 & 0.91 & $13.69-17.31$ & & & \\
\hline HCT (\%) & 168 & 0.016 & 7.81 & & & & 45.8 & 3.9 & $40.84-51.43$ \\
\hline $\operatorname{MCV}(\mathrm{fl})$ & 168 & 0.631 & 10.39 & 86.72 & 3.22 & $80.27-93.17$ & & & \\
\hline $\mathrm{MCH}(\mathrm{pg})$ & 168 & 0.510 & 1.35 & 29.07 & 1.16 & 26.75-31.39 & & & \\
\hline $\mathrm{MCHC}(\mathrm{g} / \mathrm{dl})$ & 168 & 0.002 & 0.96 & & & & 33.5 & 1.1 & $31.65-36.00$ \\
\hline $\mathrm{PLT}^{\dagger}$ & 168 & 0.230 & 3336.73 & 291.94 & 57.76 & $176.41-407.47$ & & & \\
\hline RDW-SD (fl) & 168 & 0.047 & 5.78 & & & & 39.8 & 3.17 & $35.45-45.26$ \\
\hline RDW-CV (\%) & 168 & $<0.0001$ & 0.61 & & & & 12.5 & 1.07 & $11.42-14.60$ \\
\hline PDW (fl) & 168 & $<0.0001$ & 2.84 & & & & 11.85 & 2.18 & $9.22-15.41$ \\
\hline MPV (fl) & 168 & 0.039 & 0.68 & & & & 10.25 & 1.1 & $8.82-12.00$ \\
\hline P-LCR (\%) & 168 & 0.070 & 43.55 & 26.86 & 6.60 & $13.66-40.06$ & & & \\
\hline PCT (\%) & 168 & 0.057 & 0.002 & 0.29 & 0.05 & $0.19-0.39$ & & & \\
\hline Neutrophils ${ }^{\dagger}$ & 168 & 0.007 & 1.18 & & & & 3.94 & 1.54 & $2.07-6.80$ \\
\hline Neutrophils (\%) & 168 & 0.071 & 58.68 & 54.65 & 7.66 & 39.33-69.97 & & & \\
\hline Lymphocytes $^{\dagger}$ & 168 & $<0.0001$ & 0.41 & & & & 2.31 & 0.76 & $1.42-4.04$ \\
\hline Lymphocytes (\%) & 168 & 0.257 & 49.31 & 33.47 & 7.02 & $19.43-47.51$ & & & \\
\hline Monocytes $^{\dagger}$ & 168 & $<0.0001$ & 0.03 & & & & 0.57 & 0.22 & $0.35-1.04$ \\
\hline Monocytes (\%) & 168 & 0.007 & 3.20 & & & & 7.9 & 2 & $4.87-12.09$ \\
\hline Eosinophils $^{\dagger}$ & 168 & $<0.0001$ & 0.04 & & & & 0.16 & 0.19 & $0.04-0.81$ \\
\hline Eosinophils (\%) & 168 & $<0.0001$ & 6.26 & & & & 2.3 & 2.48 & $0.62-10.34$ \\
\hline Basophils $^{\dagger}$ & 168 & $<0.0001$ & 0.001 & & & & 0.04 & 0.03 & $0.02-0.11$ \\
\hline Basophils (\%) & 168 & $<0.0001$ & 0.081 & & & & 0.7 & 0.3 & $0.22-1.3$ \\
\hline $\mathrm{IG}^{\dagger}$ & 168 & $<0.0001$ & 0.0001 & & & & 0.05 & 0.028 & $0.01-0.15$ \\
\hline IG (\%) & 168 & 0.001 & 0.00 & & & & 0.007 & 0.003 & $0.00-0.02$ \\
\hline RET (\%) & 149 & 0.002 & 0.18 & & & & 1.46 & 0.54 & $0.75-2.63$ \\
\hline RET§ & 149 & 0.002 & 0.001 & & & & 0.08 & 0.003 & $0.04-0.1325$ \\
\hline IRF (\%) & 149 & 0.0004 & 10.91 & & & & 9.6 & 4.6 & $4.98-18.33$ \\
\hline LFR (\%) & 149 & 0.0004 & 10.91 & & & & 90.4 & 4.6 & 81.68-95.03 \\
\hline MFR (\%) & 149 & 0.024 & 6.71 & & & & 8.5 & 3.75 & $4.63-14.13$ \\
\hline HFR (\%) & 149 & $<0.0001$ & 0.99 & & & & 1.0 & 0.9 & $0.20-4.43$ \\
\hline RET-He (pg) & 149 & $<0.0001$ & 2.54 & & & & 31.9 & 1.6 & $26.13-33.93$ \\
\hline IPF (\%) & 149 & $<0.0001$ & 5.65 & & & & 3.95 & 2.88 & $1.18-10.38$ \\
\hline
\end{tabular}

WBC: White Blood Cell Count; RBC: Red Blood Cell Count; HGB: Haemoglobin; HCT: Haematocrit; MCV: Mean Cell Volume; $\mathrm{MCH}$ : Mean Cell Haemoglobin; MCHC: Mean Cell Haemoglobin Concentration; PLT: Platelets; RDW CV: Red Cell Distribution (CV); RDW SD: Red Cell Distribution (SD); PDW: Platelet Distribution Width; MPV: Mean Platelet Volume; P-LCR: Platelet Large Cell Ratio; PCT: Plateletcrit; NRBC: Nucleated Red Blood Cell; IG: Immature Granulocytes; RET: Reticulocytes; IRF: Immature Reticulocyte Fraction; LFR: Low Fluorescence Reticulocytes; MFR: Moderate Fluorescence Reticulocytes; HFR: High Fluorescence Reticulocytes; RET-He: Reticulocyte Haemoglobin Equivalent; IPF: Immature Platelet Fraction.

${ }^{\dagger}$ Absolute count $\left(\mathrm{X} 10^{9} / \mathrm{l}\right)$; ${ }^{\ddagger}$ Absolute count $\left(\mathrm{X} 10^{12} / \mathrm{l}\right)$; ${ }^{\S}$ Absolute count $\left(\mathrm{X} 10^{6} / \mu \mathrm{l}\right)$ 
were calculated using the non-parametrical method. Outliers were checked and eliminated using statistical software as suggested by Turkey. The test parameters from male individuals were compared with those from female individuals using Mann-Whitney $U$ test, depending on the pattern of distribution. All results were considered significant at $p<0.05$.

\section{Results}

A total of 390 healthy subjects, comprising 168 males

Table 2: Reference ranges for parameters reported on Sysmex XN-3000 (Female, $N=222$ ).

\begin{tabular}{|c|c|c|c|c|c|c|c|c|c|}
\hline \multirow[b]{2}{*}{ Parameters } & \multirow[b]{2}{*}{$\mathbf{n}$} & \multicolumn{8}{|c|}{ Shapiro-Wilk test for normality } \\
\hline & & p-value & Variance & Mean & SD & $\begin{array}{l}\text { Range } \\
\text { (mean } \pm 2 \\
\text { SD) }\end{array}$ & Median & IQR & $\begin{array}{l}\text { Range } \\
\left(2.5^{\text {th }}-97.5^{\text {th }}\right. \\
\text { percentiles })\end{array}$ \\
\hline $\mathrm{WBC}^{\dagger}$ & 222 & $<0.0001$ & 3.64 & & & & 7.32 & 2.42 & $4.69-12.01$ \\
\hline $\mathrm{RBC}^{\ddagger}$ & 222 & 0.3196 & 0.13 & 4.66 & 0.36 & $3.95-5.38$ & & & \\
\hline HGB (g/dl) & 222 & 0.5195 & 1.11 & 13.12 & 1.05 & $11.02-15.22$ & & & \\
\hline $\mathrm{HCT}(\%)$ & 222 & 0.5032 & 8.77 & 39.85 & 2.96 & $33.93-45.77$ & & & \\
\hline $\operatorname{MCV}(f l)$ & 222 & 0.0683 & 22.33 & 85.63 & 4.73 & 76.18-95.08 & & & \\
\hline $\mathrm{MCH}(\mathrm{pg})$ & 222 & 0.0002 & 3.57 & & & & 28.5 & 2.2 & 23.53-31.34 \\
\hline $\mathrm{MCHC}(\mathrm{g} / \mathrm{dl})$ & 222 & $<0.0001$ & 0.90 & & & & 32.9 & 1.1 & $31.06-35.04$ \\
\hline $\mathrm{PLT}^{\dagger}$ & 222 & 0.1308 & 3847.72 & 308.20 & 62.03 & $184.14-432.26$ & & & \\
\hline RDW-SD (fl) & 222 & 0.001 & 8.64 & & & & 41.0 & 3.62 & $36.16-48.1$ \\
\hline RDW-CV (\%) & 222 & $<0.0001$ & 1.21 & & & & 13.0 & 1.3 & $11.76-16.54$ \\
\hline PDW (fl) & 222 & $<0.0001$ & 3.14 & & & & 11.7 & 2.2 & $9.26-16.24$ \\
\hline MPV (\%) & 222 & 0.0013 & 0.69 & & & & 10.3 & 1.1 & $9.0-12.34$ \\
\hline P-LCR (\%) & 222 & 0.0035 & 47.56 & & & & 27 & 9.38 & $16.1-43.95$ \\
\hline PCT (\%) & 222 & 0.0184 & 0.004 & & & & 0.32 & 0.07 & $0.21-0.47$ \\
\hline Neutrophils ${ }^{\dagger}$ & 222 & $<0.0001$ & 2.67 & & & & 4.09 & 2.05 & $2.24-8.40$ \\
\hline Neutrophils (\%) & 222 & 0.0109 & 79.87 & & & & 57.9 & 13.32 & $43.12-77.41$ \\
\hline Lymphocytes $^{\dagger}$ & 222 & 0.0002 & 0.40 & & & & 2.32 & 0.77 & $1.21-3.88$ \\
\hline Lymphocytes (\%) & 222 & 0.0691 & 59.60 & 31.96 & 7.72 & $16.52-47.40$ & & & \\
\hline Monocytes $^{\dagger}$ & 222 & $<0.0001$ & 0.02 & & & & 0.48 & 0.15 & $0.23-0.89$ \\
\hline Monocytes (\%) & 222 & 0.0006 & 2.83 & & & & 6.6 & 2.2 & $3.9-10.37$ \\
\hline Eosinophils $^{\dagger}$ & 222 & $<0.0001$ & 0.02 & & & & 0.14 & 0.14 & $0.03-0.54$ \\
\hline Eosinophils (\%) & 222 & $<0.0001$ & 3.22 & & & & 1.8 & 1.8 & $0.4-7.03$ \\
\hline Basophils $^{\dagger}$ & 222 & $<0.0001$ & 0.0004 & & & & 0.04 & 0.02 & $0.01-0.1$ \\
\hline Basophils (\%) & 222 & $<0.0001$ & 0.09 & & & & 0.5 & 0.3 & $0.2-1.5$ \\
\hline $\mathrm{IG}^{\dagger}$ & 222 & $<0.0001$ & 0.0012 & & & & 0.05 & 0.05 & $0.01-0.14$ \\
\hline IG (\%) & 222 & 0.0182 & 0.00 & & & & 0.007 & 0.004 & $0-0.02$ \\
\hline RET (\%) & 209 & 0.0186 & 0.15 & & & & 1.33 & 0.51 & $0.71-2.31$ \\
\hline RET§ & 209 & $<0.0001$ & 0.00 & & & & 0.06 & 0.004 & $0.03-0.1075$ \\
\hline IRF (\%) & 209 & $<0.0001$ & 14.71 & & & & 8.9 & 5.2 & $3.64-19.43$ \\
\hline LFR (\%) & 209 & $<0.0001$ & 14.71 & & & & 91.1 & 5.2 & 80.58-96.38 \\
\hline MFR (\%) & 209 & 0.0745 & 7.7 & 8.29 & 2.77 & $2.75-13.84$ & & & \\
\hline HFR (\%) & 209 & $<0.0001$ & 1.94 & & & & 0.9 & 0.9 & $0-5.05$ \\
\hline RET-He (pg) & 209 & $<0.0001$ & 4.76 & & & & 31.0 & 2.8 & $24.78-33.80$ \\
\hline IPF (\%) & 209 & $<0.0001$ & 6.07 & & & & 3.50 & 2.7 & $1.23-10.58$ \\
\hline
\end{tabular}

WBC: White Blood Cell Count; RBC: Red Blood Cell Count; HGB: Haemoglobin; HCT: Haematocrit; MCV: Mean Cell Volume; $\mathrm{MCH}$ : Mean Cell Haemoglobin; MCHC: Mean Cell Haemoglobin Concentration; PLT: Platelets; RDW CV: Red Cell Distribution (CV); RDW SD: Red Cell Distribution (SD); PDW: Platelet Distribution Width; MPV: Mean Platelet Volume; P-LCR: Platelet Large Cell Ratio; PCT: Plateletcrit; NRBC: Nucleated Red Blood Cell; IG: Immature Granulocytes; RET: Reticulocytes; IRF: Immature Reticulocyte Fraction; LFR: Low Fluorescence Reticulocytes; MFR: Moderate Fluorescence Reticulocytes; HFR: High Fluorescence Reticulocytes; RET-He: Reticulocyte Haemoglobin Equivalent; IPF: Immature Platelet Fraction.

${ }^{\dagger}$ Absolute count $\left(\mathrm{X} 10^{9} / \mathrm{l}\right)$; ${ }^{\ddagger}$ Absolute count $\left(\mathrm{X} 10^{12} / \mathrm{l}\right)$; ${ }^{\S}$ Absolute count $\left(\mathrm{X} 10^{6} / \mu \mathrm{l}\right)$ 
and 222 female adult volunteers were enrolled for calculating the reference ranges of full blood count on Sysmex XN-3000 and XE-5000 respectively. Descriptive statistics of the haematological reference ranges for healthy adults in the Malaysian population are shown in Table 1 and Table 2. Shapiro-Wilk test was used to analyse the distribution for all parameters. When data were normally distributed $(p<0.05)$, statistical parameters such as mean, SD, and range (mean \pm 2SD) were calculated and reported. When parameters displayed a skewed distribution ( $p>0.05$ ), median and IQR with percentile based on Range (2.5-97.5 percentile) were considered. Table 1 and Table 2 show the normal range values of all the $\mathrm{XN}-3000$ parameters for males and females respectively. Normal ranges of all the XE-5000 parameters for male and female subjects are shown in Supplementary Table 1 and Supplemental Table 2. There are no nucleated red blood cells (nRBC) detected on both analysers and this finding is consistent with our subjects being healthy volunteers. A comparison of the reference ranges between male and female was made for the two analysers showing a significant difference between genders (Table 3). For all parameters tested, less than or equal to $0.5 \%$ carry-over

Table 3: Reference ranges of male and female measured in Sysmex XN-3000 and XE-5000.

\begin{tabular}{|c|c|c|c|c|c|}
\hline \multicolumn{2}{|l|}{ Parameters } & \multirow{3}{*}{$\begin{array}{l}\text { XN-3000 } \\
\mathbf{( 2 . 5 ^ { \text { th } } - 9 7 . 5 ^ { \text { th } }} \text { percentiles) } \\
4.34-10.15^{\pi} \\
4.69-12.01\end{array}$} & \multirow{3}{*}{$\begin{array}{l}\text { p-value } \\
0.2254\end{array}$} & \multirow{3}{*}{$\begin{array}{l}\text { XE-5000 } \\
\left(2.5^{\text {th }}-97.5^{\text {th }} \text { percentiles) }\right. \\
4.33-10.07^{\text {T }} \\
4.60-11.91 \\
\end{array}$} & \multirow{3}{*}{$\begin{array}{l}\text { p-value } \\
0.3317\end{array}$} \\
\hline \multirow{2}{*}{$\mathrm{WBC}^{\dagger}$} & $M$ & & & & \\
\hline & $\mathrm{F}$ & & & & \\
\hline \multirow{2}{*}{$\mathrm{RBC}^{\ddagger}$} & $M$ & $4.64-6.03^{\pi}$ & \multirow{2}{*}{$<0.0001$} & $4.70-5.96 \pi$ & \multirow{2}{*}{$<0.0001$} \\
\hline & $\mathrm{F}$ & $3.95-5.38 \pi$ & & $4.01-5.34 \pi$ & \\
\hline \multirow{2}{*}{$\mathrm{HGB}(\mathrm{g} / \mathrm{dl})$} & M & $13.69-17.31^{\pi}$ & \multirow{2}{*}{$<0.0001$} & $13.48-17.08^{\pi}$ & \multirow{2}{*}{$<0.0001$} \\
\hline & $\mathrm{F}$ & $11.02-15.22^{\pi}$ & & $11.03-14.95^{\pi}$ & \\
\hline \multirow{2}{*}{$\mathrm{HCT}(\%)$} & $M$ & $40.84-51.43$ & \multirow{2}{*}{$<0.0001$} & $41.2-50.58$ & \multirow{2}{*}{$<0.0001$} \\
\hline & $\mathrm{F}$ & $33.93-45.77^{\pi}$ & & $34.57-45.46 \pi$ & \\
\hline \multirow{2}{*}{$\operatorname{MCV}(f l)$} & M & $80.27-93.17^{\pi}$ & \multirow{2}{*}{0.0353} & $80.33-92.61$ & \multirow{2}{*}{0.1469} \\
\hline & $\mathrm{F}$ & $76.18-95.08 \pi$ & & $75.46-93.54 \pi$ & \\
\hline \multirow{2}{*}{$\mathrm{MCH}(\mathrm{pg})$} & $M$ & $26.75-31.39 \pi$ & \multirow{2}{*}{$<0.0001$} & $26.5-30.88 \pi$ & \multirow{2}{*}{$<0.0001$} \\
\hline & $\mathrm{F}$ & $23.53-31.34$ & & $23.76-30.73$ & \\
\hline \multirow{2}{*}{$\mathrm{MCHC}(\mathrm{g} / \mathrm{dl})$} & M & $31.65-36$ & \multirow{2}{*}{$<0.0001$} & $31.45-35.7$ & \multirow{2}{*}{$<0.0001$} \\
\hline & $\mathrm{F}$ & $31.06-35.04$ & & $30.6-34.14$ & \\
\hline \multirow{2}{*}{$\mathrm{PLT}^{\dagger}$} & M & $176.41-407.47^{\Uparrow}$ & \multirow{2}{*}{0.0148} & $182.9-389.1^{\pi}$ & \multirow{2}{*}{$<0.0001$} \\
\hline & $\mathrm{F}$ & $184.14-432.26^{\pi}$ & & $192.46-435.94^{\pi}$ & \\
\hline \multirow{2}{*}{ RDW-SD (fl) } & $M$ & $35.45-45.26$ & \multirow{2}{*}{$<0.0001$} & $36.69-46.19^{\pi}$ & \\
\hline & $\mathrm{F}$ & $36.16-48.1$ & & $37.86-49.24$ & $<0.0001$ \\
\hline & $M$ & $11.42-14.6$ & & $12-14.9$ & \\
\hline RDVV-CV (\%) & $\mathrm{F}$ & $11.76-16.54$ & $<0.0001$ & $12.3-16.73$ & $<0.0001$ \\
\hline & $M$ & $9.22-15.41$ & 09548 & 9.5-15.97 & 02634 \\
\hline PDVV (TI) & $\mathrm{F}$ & $9.26-16.24$ & 0.9548 & $9.5-15.74$ & 0.2634 \\
\hline & $M$ & $8.82-12$ & 00503 & $8.9-11.89$ & 08573 \\
\hline MPV (\%) & $\mathrm{F}$ & $9-12.34$ & 0.0503 & $8.9-12.04$ & 0.8513 \\
\hline & $M$ & $13.66-40.06 \pi$ & & $13.92-39.6 \pi$ & \\
\hline P-LCR (\%) & $\mathrm{F}$ & $16.1-43.95$ & 0.1428 & $15.37-41.81$ & 0.9841 \\
\hline PCT (\%) & $M$ & $0.19-0.39 \pi$ & & $0.19-0.39 \pi$ & \\
\hline PCl (\%) & $\mathrm{F}$ & $0.21-0.47$ & $<0.0001$ & $0.21-0.45$ & $<0.0001$ \\
\hline & $M$ & $2.07-6.8$ & & $2.18-6.84$ & \\
\hline Neutrophils ${ }^{\top}$ & $\mathrm{F}$ & $2.24-8.4$ & 0.0083 & $2.37-8.34$ & 0.0162 \\
\hline & $M$ & $39.33-69.97^{\pi}$ & & $41.37-70.93^{\pi}$ & $<0 \cap 0 \cap 1$ \\
\hline Neutropnils (\%) & $\mathrm{F}$ & $43.12-77.41$ & 0.0001 & 45.33-77.39 & $<0.0001$ \\
\hline J vmphocytes† & $M$ & $1.42-4.04$ & 07904 & 1.34-3.8 & 05579 \\
\hline Lympnocyles' & $\mathrm{F}$ & $1.21-3.88$ & 0.1904 & $1.23-3.67$ & 0.0519 \\
\hline
\end{tabular}




\begin{tabular}{|c|c|c|c|c|c|}
\hline \multirow{2}{*}{ Lymphocytes (\%) } & $M$ & $19.43-47.51^{\pi}$ & \multirow{2}{*}{0.0823} & 18.87-45.91 & \multirow{2}{*}{0.0574} \\
\hline & $\mathrm{F}$ & $16.52-47.40^{\pi}$ & & 16.01-45.89ा & \\
\hline \multirow{2}{*}{ Monocytes $^{\dagger}$} & $M$ & $0.35-1.04$ & \multirow{2}{*}{$<0.0001$} & $0.32-0.93$ & \multirow{2}{*}{$<0.0001$} \\
\hline & $\mathrm{F}$ & $0.23-0.89$ & & $0.23-0.85$ & \\
\hline \multirow{2}{*}{ Monocytes (\%) } & $M$ & $4.87-12.09$ & \multirow{2}{*}{$<0.0001$} & $4.45-11.7$ & \multirow{2}{*}{$<0.0001$} \\
\hline & $\mathrm{F}$ & $3.9-10.37$ & & $3.8-10.3$ & \\
\hline \multirow{2}{*}{ Eosinophils $^{\dagger}$} & $M$ & $0.04-0.81$ & \multirow{2}{*}{0.0207} & $0.05-0.9$ & \multirow{2}{*}{0.0061} \\
\hline & $\mathrm{F}$ & $0.03-0.54$ & & $0.03-0.52$ & \\
\hline \multirow{2}{*}{ Eosinophils (\%) } & $M$ & $0.62-10.34$ & \multirow{2}{*}{0.0009} & $0.7-11.5$ & \multirow{2}{*}{0.0002} \\
\hline & $\mathrm{F}$ & $0.4-7.03$ & & $0.42-6.83$ & \\
\hline \multirow{2}{*}{ Basophils $^{\dagger}$} & $M$ & $0.02-0.11$ & \multirow{2}{*}{0.0015} & $0.01-0.08$ & \multirow{2}{*}{0.0138} \\
\hline & $\mathrm{F}$ & $0.01-0.1$ & & $0.01-0.07$ & \\
\hline \multirow{2}{*}{ Basophils (\%) } & $M$ & $0.22-1.3$ & \multirow{2}{*}{$<0.0001$} & $0.1-1$ & \multirow{2}{*}{0.0030} \\
\hline & $\mathrm{F}$ & $0.2-1.5$ & & $0.06-0.99$ & \\
\hline \multirow{2}{*}{$\mathrm{IG}^{\dagger}$} & $M$ & $0.01-0.15$ & \multirow{2}{*}{0.3932} & $0-0.07$ & \multirow{2}{*}{0.0406} \\
\hline & $\mathrm{F}$ & $0.01-0.14$ & & $0-0.05$ & \\
\hline \multirow{2}{*}{ IG (\%) } & $M$ & $0.00-0.02$ & \multirow{2}{*}{0.0529} & $0-0.01$ & \multirow{2}{*}{0.0062} \\
\hline & $\mathrm{F}$ & $0.00-0.02$ & & $0-0.01$ & \\
\hline \multirow{2}{*}{ RET (\%) } & $M$ & $0.75-2.63$ & \multirow{2}{*}{0.0058} & $0.52-2.43$ & רחמחם \\
\hline & $\mathrm{F}$ & $0.71-2.31$ & & $0.47-2.03$ & 0.0002 \\
\hline RFT\#§ & $M$ & $0.04-0.1325$ & $<00001$ & $0.03-0.1278$ & $<0 \cap 0 \cap 1$ \\
\hline Rᄃt" & $\mathrm{F}$ & $0.03-0.1075$ & -0.0001 & $0.02-0.09$ & -0.0001 \\
\hline & $M$ & $4.98-18.33$ & & $0.5-8.16$ & \\
\hline $\mathrm{IRF}(\%)$ & $\mathrm{F}$ & $3.64-19.43$ & 0.1428 & $0.6-12.34$ & 0.9523 \\
\hline IFR (\%) & $M$ & $81.68-95.03$ & & $91.85-99.5$ & 09523 \\
\hline LFR (\%) & $\mathrm{F}$ & $80.58-96.38$ & 0.1428 & $87.66-99.4$ & 0.9523 \\
\hline MFR (\%) & $M$ & $4.63-14.13$ & 01747 & $0.5-7.98$ & 09653 \\
\hline IVIFR (\%) & $\mathrm{F}$ & $2.75-13.84 \pi$ & 0.1741 & $0.6-10.51$ & 0.9653 \\
\hline & $M$ & $0.2-4.43$ & & $0-1$ & 0163 \\
\hline HFR (\%) & $\mathrm{F}$ & $0-5.05$ & 0.1362 & $0-1.14$ & 0.5103 \\
\hline & $M$ & $26.13-33.93$ & & 28.7-37.96 & \\
\hline REl-He (pg) & $\mathrm{F}$ & $24.78-33.8$ & $<0.0001$ & 23.79-38.34 & $<0.0001$ \\
\hline IPF (\%) & $M$ & $1.18-10.38$ & 01382 & $0.4-2.96$ & 00162 \\
\hline IFF (\%) & $\mathrm{F}$ & $1.23-10.58$ & 0.1382 & $0.3-3.1$ & 0.0102 \\
\hline
\end{tabular}

Median with range $\left(2.5^{\text {th }}-97.5^{\text {th }}\right.$ percentiles $) .{ }^{\dagger}$ Absolute count $\left(\mathrm{X} 10^{9} / \mathrm{l}\right) ;{ }^{\ddagger}$ Absolute count $\left(\mathrm{X} 10^{12} / \mathrm{l}\right) ;{ }^{\S} \mathrm{Absolute}$ count $\left(\mathrm{X} 10^{6} / \mu \mathrm{l}\right) ;{ }^{\mathrm{T}} \mathrm{Mean}$ $\pm 2 \mathrm{SD}$

Table 4: Comparison between Sysmex XN-3000 and XE-5000.

\begin{tabular}{|l|l|l|l|}
\hline \multirow{2}{*}{ Parameters } & Correlation, $\mathbf{r}(\mathbf{9 5} \% \mathrm{Cl})$ & \multicolumn{2}{|c|}{ Median (range) } \\
\cline { 3 - 4 } & $0.9792(0.9745$ to 0.9830$)$ & $7.29(4.74-11.31)$ & $7.12(4.6-11.14)$ \\
\hline WBC $^{\dagger}$ & $0.9886(0.9860$ to 0.9907$)$ & $4.91(4.11-5.83)$ & $4.93(4.15-5.75)$ \\
\hline RBC $^{\ddagger}$ & $0.9888(0.9863$ to 0.9909$)$ & $14.15(11.07-17.23)$ & $13.97(11.02-16.92)$ \\
\hline HGB $(\mathrm{g} / \mathrm{dl})$ & $0.9821(0.9780$ to 0.9854$)$ & $42.6(34.02-51.18)$ & $42.6(34.63-50.57)$ \\
\hline HCT $(\%)$ & $0.9139(0.8952$ to 0.9293$)$ & $86.5(76.46-94.25)$ & $86.35(77.28-92.82)$ \\
\hline MCV $(f l)$ & $0.9234(0.9067$ to 0.9371$)$ & $28.8(24.83-31.32)$ & $28.4(24.51-30.82)$ \\
\hline MCH $(\mathrm{pg})$ & $0.8513(0.8205$ to 0.8772$)$ & $33.2(31.3-35.45)$ & $32.75(30.9-35.01)$ \\
\hline MCHC $(\mathrm{g} / \mathrm{dl})$ & $0.9547(0.9447$ to 0.9630$)$ & $297(190.6-433)$ & $301(193.8-430.4)$ \\
\hline PLT ${ }^{\dagger}$ & $0.9389(0.9255$ to 0.9500$)$ & $40.3(35.98-47.17)$ & $41.9(37.7-48.54)$ \\
\hline RDW-SD (fl) & $0.9781(0.9731$ to 0.9821$)$ & $12.8(11.6-15.9)$ & $13.3(12.1-16.12)$ \\
\hline RDW-CV $(\%)$ & & & \\
\hline
\end{tabular}




\begin{tabular}{|c|c|c|c|}
\hline PDW (fl) & 0.9384 (0.9249 to 0.9496$)$ & $11.8(9.28-15.75)$ & $11.85(9.5-15.72)$ \\
\hline MPV (\%) & $0.9528(0.9424$ to 0.9614$)$ & $10.3(8.9-12.22)$ & $10.2(8.9-12)$ \\
\hline P-LCR (\%) & 0.9661 (0.9585 to 0.9723$)$ & $27(15.68-43.15)$ & $26.4(15.3-41.6)$ \\
\hline PCT (\%) & 0.964 (0.9560 to 0.9706$)$ & $0.31(0.21-0.44)$ & $0.3(0.21-0.43)$ \\
\hline Neutrophils ${ }^{\dagger}$ & $0.9824(0.9784$ to 0.9856$)$ & $3.98(2.22-7.74)$ & $4.1(2.3-7.68)$ \\
\hline Neutrophils (\%) & $0.9899(0.9876$ to 0.9917$)$ & 56.35 (41.88-76.19) & $57.9(44.16-76.45)$ \\
\hline Lymphocytes $^{\dagger}$ & $0.9783(0.9734$ to 0.9823$)$ & $2.31(1.34-3.93)$ & $2.21(1.27-3.74)$ \\
\hline Lymphocytes (\%) & $0.9857(0.9825$ to 0.9884$)$ & $32.7(16.08-45.72)$ & $31.8(15.63-44.6)$ \\
\hline Monocytes $^{\dagger}$ & 0.8836 (0.8589 to 0.9042$)$ & $0.52(0.3-0.91)$ & $0.5(0.26-0.87)$ \\
\hline Monocytes (\%) & 0.8674 (0.8396 to 0.8907$)$ & $7.3(4.1-11.4)$ & $7(3.9-10.9)$ \\
\hline Eosinophils $^{\dagger}$ & $0.9773(0.9721$ to 0.9814$)$ & $0.15(0.04-0.64)$ & $0.15(0.03-0.65)$ \\
\hline Eosinophils (\%) & 0.9731 (0.9670 to 0.9780$)$ & $2(0.5-8.51)$ & $2.1(0.5-8.2)$ \\
\hline Basophils $^{\dagger}$ & $0.7566(0.7092$ to 0.7972$)$ & $0.04(0.02-0.11)$ & $0.02(0.01-0.07)$ \\
\hline Basophils (\%) & 0.7664 (0.7206 to 0.8056$)$ & $0.6(0.2-1.42)$ & $0.3(0.1-1)$ \\
\hline $\mathrm{IG}^{\dagger}$ & $0.5517(0.4762$ to 0.6191$)$ & $0.05(0.01-0.14)$ & $0.01(0-0.07)$ \\
\hline IG (\%) & $0.4367(0.01693$ to 0.7256$)$ & $0.01(0-0.01)$ & $0(0-0.01)$ \\
\hline RET (\%) & 0.9275 (0.9109 to 0.9411$)$ & $1.36(0.74-2.38)$ & $1.2(0.51-2.24)$ \\
\hline RET§ & $0.924(0.9067$ to 0.9382$)$ & $0.07(0.03-0.12)$ & $0.06(0.03-0.11)$ \\
\hline IRF (\%) & 0.6978 (0.6385 to 0.7488$)$ & $9.3(4.2-18.61)$ & $3(0.58-9.55)$ \\
\hline $\operatorname{LFR}(\%)$ & $0.6978(0.6385$ to 0.7488$)$ & $90.7(81.39-95.81)$ & 97 (90.46-99.42) \\
\hline MFR (\%) & 0.6491 (0.5827 to 0.7069$)$ & $8.2(3.9-14.51)$ & $2.85(0.58-8.79)$ \\
\hline HFR (\%) & $0.4888(0.4030$ to 0.5661$)$ & $1(0-4.51)$ & $0.1(0-1.1)$ \\
\hline RET-He (pg) & $0.832(0.7959$ to 0.8622$)$ & $31.4(25.1-33.8)$ & $34.85(25.29-38.02)$ \\
\hline IPF(\%) & 0.6749 (0.6121 to 0.7292$)$ & $3.7(1.2-10.51)$ & $1(0.3-3)$ \\
\hline
\end{tabular}

Median with range $\left(2.5^{\text {th }}-97.5^{\text {th }}\right.$ percentiles $) ;{ }^{\dagger}$ Absolute count $\left(\mathrm{X} 10^{9} / \mathrm{l}\right) ;{ }^{\ddagger}$ Absolute count $\left(\mathrm{X} 10^{12} / \mathrm{l}\right) ;{ }^{\S} \mathrm{Absolute}$ count $\left(\mathrm{X} 10^{6} / \mu \mathrm{l}\right)$

was seen. Comparison studies demonstrated strong correlations on most of the key parameters between XN-3000 and XE-5000 (Table 4 and Figure 1).

\section{Discussion}

Meaningful information that reflects patients' clinical conditions can only be provided by comparing the observed value with reference ranges [16]. Automated haematologyanalysers have been improved especiallyon the measurements of advanced clinical haematological parameters. Clinical evaluation at different timelines for diagnostic purposes, the management, monitoring, and following-up of cases are largely dependent on these parameters and the knowledge of reliable reference ranges. The Malaysian population-based reference values for FBC were previously established using a total of 1376 healthy individuals, comprised of onethird Malay, Chinese and Indian subjects, respectively. Clinically significant differences related to race were not found in any of the test parameters. However, age and gender-specific values were obtained for haemoglobin, red blood cells count, haematocrit, platelets count, and serum ferritin [10]. The principle method of detection and technology were similar on both analysers for commonly reported clinical haematological parameters, such as total white blood cell count and its differentials, haemoglobin, and red cell indices, and platelet counts. The reference ranges of these parameters showed no significant difference as expected. In the present study, we showed that the reference interval for full clinical haematological parameters in a Malaysian cohort using the $\mathrm{XN}-3000$ could be established, as transference of the ranges was done on two analysers at the time in our haematology laboratory.

The present study was not just conducted to determine the reference ranges for common full blood count parameters measured by XN-3000. We also placed emphasis on the assessment and transference of ranges of advanced clinical haematological XE-5000 to $\mathrm{XN}-3000$ for the same evaluation in the Malaysian population. These advanced parameters were meant to represent the different maturation stages of the three main haemopoeitic cell lineages being red blood cells, granulocytes, and platelets. For red blood cell maturation, reticulocytes signify the presence of newly formed and immature cells released from the bone marrow. Its presence in the circulation could signal some pathological mechanisms that have caused the bone marrow to increase red blood cell production such as blood loss and haemolysis [17]. Reticulocytes are fractionated into three categories which represent different stages of maturity depending on the intensity of fluorescence: LFR (low fluorescence reticulocytes), MFR (medium fluorescence reticulocytes), and HFR (high fluorescence reticulocytes). Immature reticulocyte 

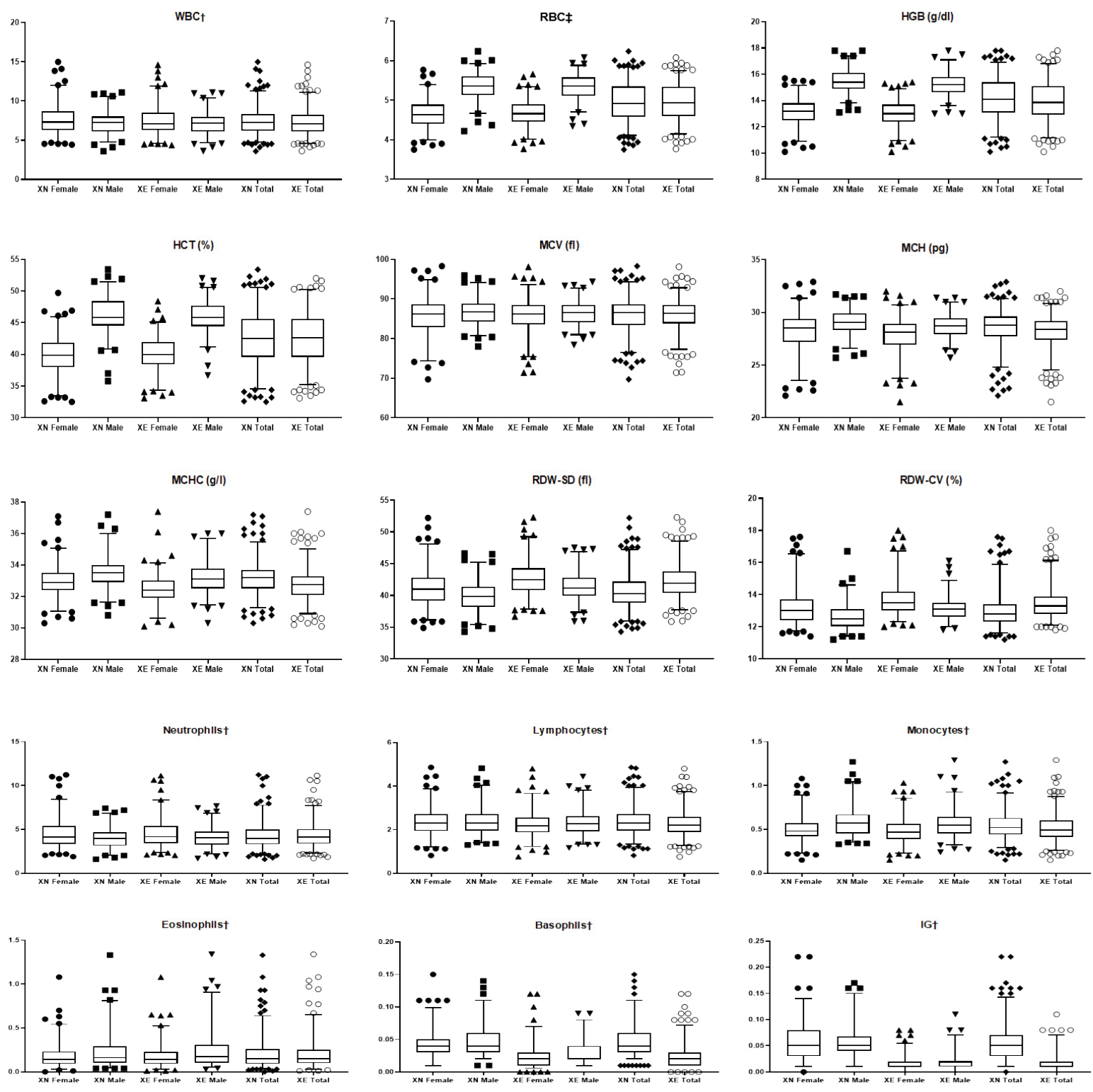

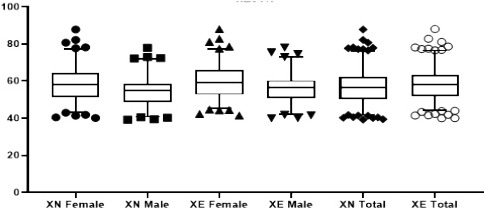
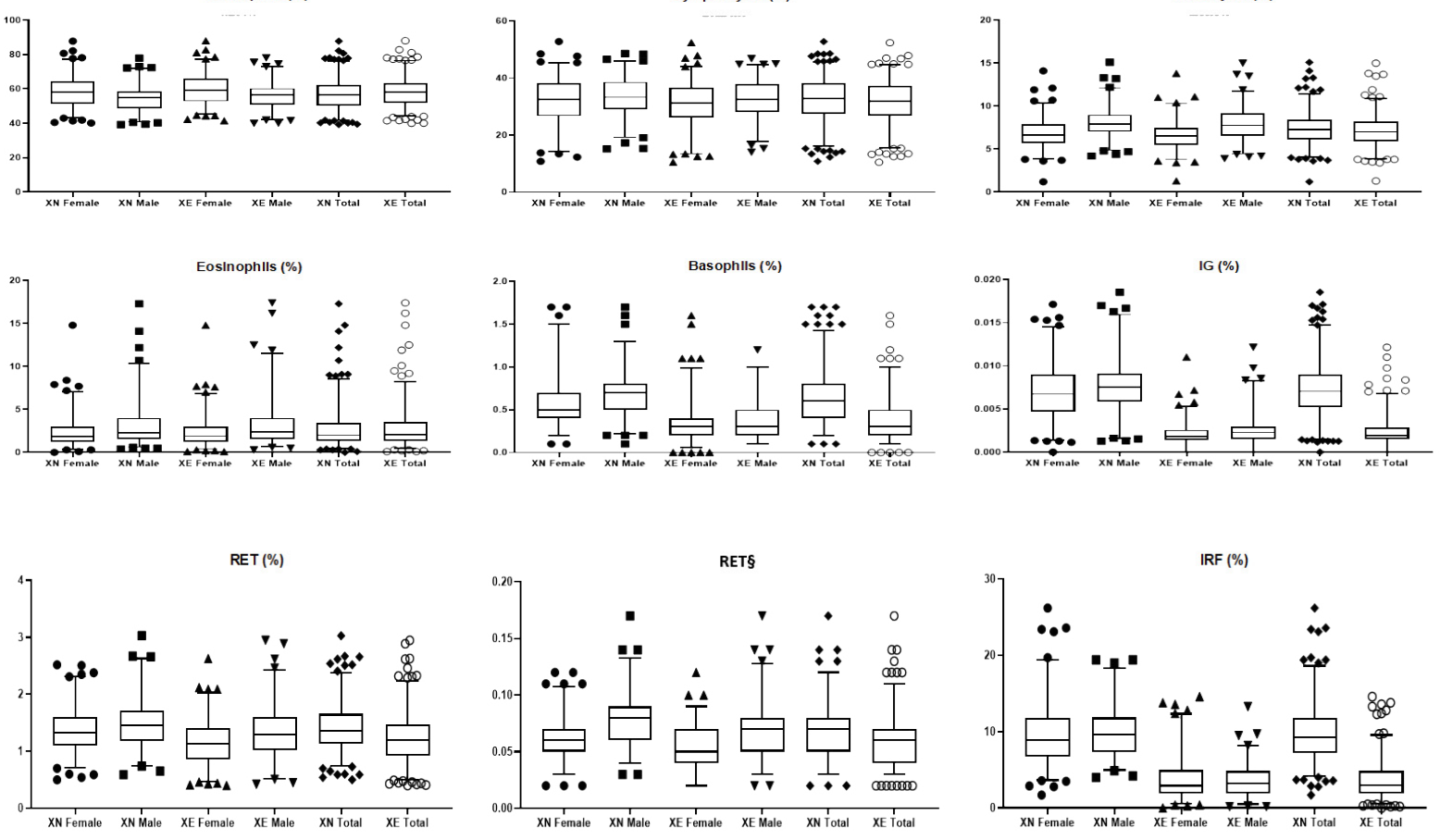

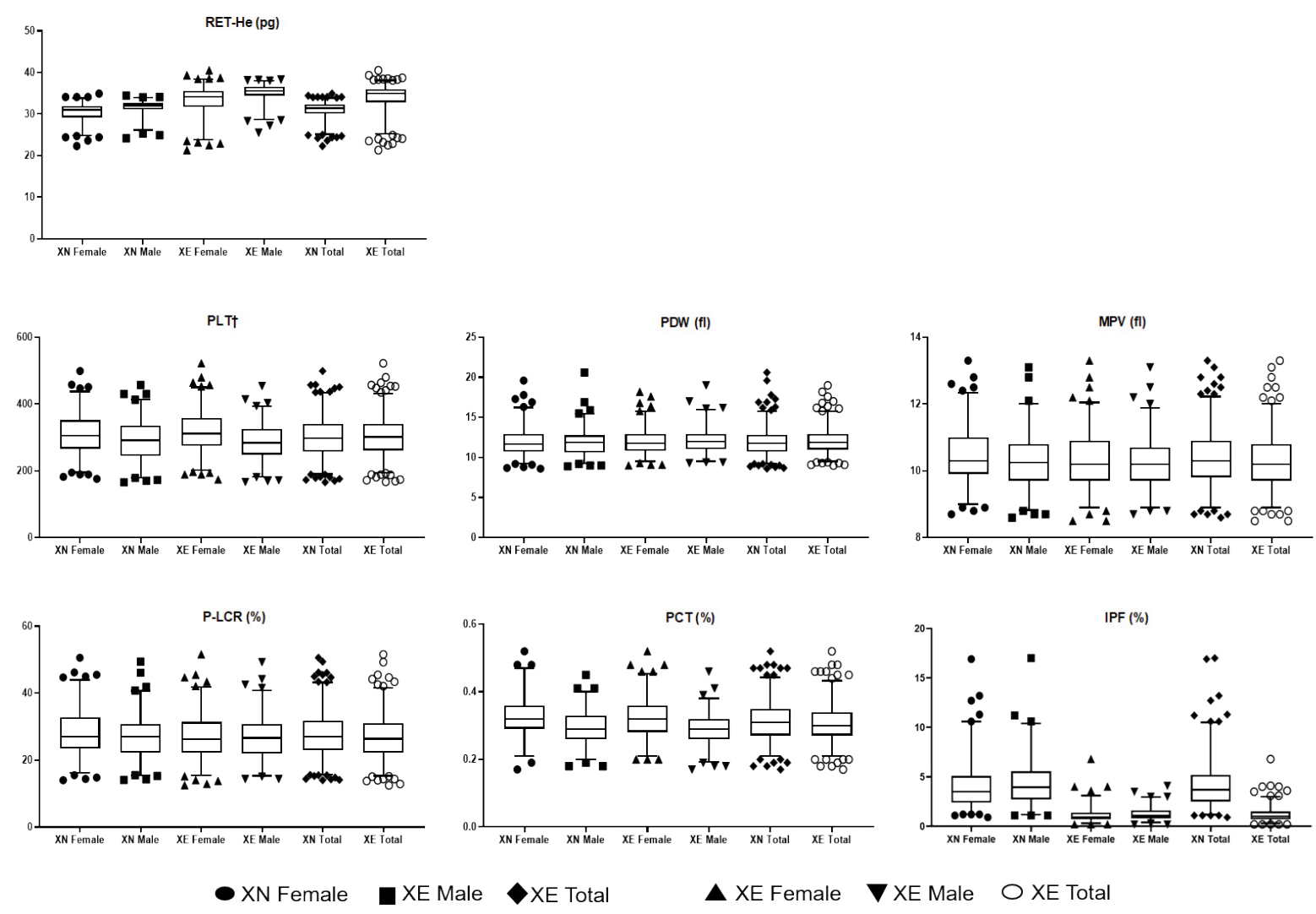

XE Total

Figure 1: Comparison of full blood count reference ranges between male and female healthy Malaysian population obtained on the Sysmex XE-5000 and XN-3000.

Box and Whiskers $\left(2.5^{\text {th }}-97.5^{\text {th }}\right.$ percentile) with the removal of outliners; ${ }^{\dagger}$ Absolute count $\left(X 10^{9} / l\right)$; ${ }^{\ddagger} A b s o l u t e ~ c o u n t ~\left(X 10^{12} / l\right)$; $\S$ Absolute count $\left(\mathrm{X} 10^{6} / \mu \mathrm{l}\right)$

fraction (IRF) provides a standardized measurement of immature reticulocytes and is calculated from the sum of MFR plus HFR. IRF plays a role as an indicator of erythropoiesis that has been proposed to correlate well with the engraftment of neutrophils as published by researchers [18]. For routine reporting of cases on $\mathrm{XN}-3000$ for the advanced clinical haematological parameters, the newer reference range of IRF was adopted. Ret-He which has been used as a surrogate to flag cases of iron deficiency $[19,20]$ showed a significant difference between male and female with a good correlation between the analysers.

Platelet counts traditionally represent the adequacy of circulating mature forms quantitatively. IPF is considered as a parameter that detects reticulated platelets or young, newly released platelets [21]. The distinction between the commonly used platelet count and IPF is that the IPF contains a higher concentration of RNA than its mature counterpart. This is meant to provide a picture of the megakaryocytic activity in the bone marrow. This parameter has been investigated in cases of bone marrow recovery after haemopoietic stem cell transplant $[22,23]$ and many other haematological disorders [24]. The range of IPF, as well as IRF, was significantly different between the two analysers (Table 4). According to Van der Linden, et al. and Ko, et al., the higher reference intervals of IPF obtained with the XN compared with the XE series may reflect the fact that both analyser shave different metrological characteristics. However, the IPF measured on the XN series appeared to perform better, showing better precision and accuracy compared to the XE series $[21,25]$. IPF which is considered the equivalent to IRF in red cells measures immature platelets. Therefore, with the XN-3000 using a novel fluorescent dye for measuring fluorescent platelets and IPF, IPF results will be more specific and this suggests the necessity of new reference intervals for IPF to be established.

The presence of the immature spectrum of granulocytic maturation is considered highly unusual and warrants further investigation. IGs include promyelocytes, myelocytes, and metamyelocytes. The availability of this parameter was meant to extend the differential count of white blood cells and to eliminate inter-observer variability on blood films while increasing the sensitivity to flag potential irregularities in the peripheral blood. IGs are detected by the $\mathrm{XN}-3000$ using the fluorescent technique. As with the extended reticulocyte parameters, a low IG value that is near zero would not show a good correlation statistically (Table 4).

In patients with haematological diseases, an assessment via bone marrow aspiration and trephine 
biopsy is an invasive method to use as a means to monitor the main haematopoietic elements; erythropoiesis, granulopoiesis, and lymphopoiesis as well as megakaryopoiesis of the bone marrow at every follow-up visit. A typical full blood count with 5 to 6 differential cell counts may be inadequate to reflect the bone marrow environment at different stages of treatment. A peripheral blood smear is subject to different preparations and inter-observer variability depending on one's experience. Therefore, surrogate markers that could reflect the haemopoietic response obtained from peripheral blood are most desirable. However, thorough and careful determination of these parameters using healthy individuals as a standard marker is pertinent to avoid misrepresentation and false-flagging events. Therefore, the next step would be to further our evaluations using peripheral blood of certain pathological states in correlation with other haematological investigations.

In conclusion, we have generated to the best of our knowledge, the first report on reference ranges in a Malaysian population using the Sysmex XN-3000 haematology analyser which has been evaluated using robust statistical analysis to ensure the safe transference of values from the XE series to ensure reliable and good clinical correlation in day-to-day practice. As a tertiary centre for haematology services in Malaysia, the XN-3000 showed good analytical performance and provided solutions for laboratories with medium to high workloads catering to the ever-clinical needs of the laboratory. The importance of population-specific read-out of haematological parameters cannot be understated. Currently, there is a need for establishing local guidelines rather than the adoption of generalised reference intervals.

\section{Funding Statement}

The study was supported by a grant awarded by the Sysmex Asia Pacific Collaborative Study. The grant was used solely for research purposes such as the acquisition of reagents and honorariums for volunteers. Sysmex Asia Pacific Pte Ltd. had no role in the study design, data acquisition, the decision to publish, or preparation of the manuscript. None of the authors were reimbursed for conducting this study.

\section{Acknowledgment}

Special thanks to Kit Yee, Cheah for helping in designing the study; Norazrina Masani and Putri Intan Hafizah Megat Mohd Azlan for assisting in data collection. The authors thank all volunteers who consented to be a part of the study, as well as the clinical and laboratory staff of the Department of Haematology, Hospital Ampang for any direct and indirect participation in the study.

\section{Authors Contribution}

Contribution: SLC designed the study, collected and analysed the data, and wrote the manuscript. $\mathrm{RH}$ collected the data; KBL analysed data and performed the statistical analysis. SMT and AWAA reviewed and edited the manuscript. All authors reviewed and approved the manuscript.

\section{Conflict of Interest}

There are no conflicts of interest declared by any of the authors.

\section{References}

1. Herklotz R, Luthi U, Ottiger C, Huber AR (2006) Metaanalysis of reference values in hematology. Ther Umsch 63: 5-24.

2. Solberg HE (1987) Approved recommendation (1987) on the theory of reference values. Part 5. Statistical treatment of collected reference values. Determination of reference limits. Clinica Chimica Acta 170: S13-S32.

3. Grasbeck R (1969) Establishment and use of normal values. Scand J Clin Invest 110: 1-15.

4. Boyd J (2010) Defining, establishing, and verifying reference intervals in the clinical laboratory; Approved Guidelines, CLSI document C28-A3.

5. Solberg H, Stamm D (1991) IFCC recommendation - theory of reference values. Part 4. Control of analytical variation in the production, transfer and application of reference values. J Automat Chem 13: 231-234.

6. Solberg HE (1987) International federation of clinical chemistry (IFCC), Scientific committee, Clinical section, Expert panel on theory of reference values, and International committee for standardization in haematology (ICSH), Standing committee on reference values. Approved Recommendation (1986) on the theory of reference values. Part 1. The concept of reference values. J Clin Chem Clin Biochem 25: 337-342.

7. PetitClerc C, Solberg HE (1987) Approved recommendation (1987) on the theory of reference values. Part 2. Selection of individuals for the production of reference values. Clinica Chimica Acta 170: S1-S11.

8. Solberg H, Petit Clerc C (1988) Approved recommendation (1988) on the theory of reference values. Part 3. Preparation of individuals and collection of specimens for the production of reference values. Clinica Chimica Acta 177: S3-S11.

9. Dybkœr R, Solberg HE (1987) Approved recommendation (1987) on the theory of reference values. Part 6. Presentation of observed values related to reference values. Clinica Chimica Acta 170: S33-S41.

10. Ambayya A, Su AT, Osman NH, Nik-Samsudin NR, Khalid $\mathrm{K}$, et al. (2014) Haematological reference intervals in a multiethnic population. PLoS One 9: e91968.

11. Hotton J, Broothaers J, Swaelens C, Cantinieaux B (2013) Performance and abnormal cell flagging comparisons of three automated blood cell counters: Cell-Dyn Sapphire, DxH-800, and XN-2000. Am J Clin Pathol 140: 845-852.

12. Briggs C, Longair I, Kumar P, Singh D, Machin SJ (2012) Performance evaluation of the Sysmex haematology $\mathrm{XN}$ modular system. J Clin Pathol 65: 1024-1030.

13. Seo JY, Lee S-T, Kim S-H (2015) Performance evaluation of the new hematology analyzer Sysmex $\mathrm{XN}$-series. Int J Lab Hematol 37: 155-164.

14. Lewis SM (2009) International council for standardization in haematology - the first 40 years. Int $\mathrm{J}$ Lab Hematol 31: 253-267. 
15. (2008) Clinical and laboratory standards institute. Defining $\mathrm{E}$, and verifying reference intervals in the clinical laboratory. Approved Guideline. ( $3^{\text {rd }}$ edn), Wayne, PA, CLSI.

16. Solberg $H$ (1993) A guide to IFCC recommendations on reference values. J Int Fed Clin Chem 5: 162-165.

17. Barcellini W, Fattizzo B (2015) Clinical applications of hemolytic markers in the differential diagnosis and management of hemolytic anemia. Dis Markers 2015: 635670.

18. Yahng SA, Lee JW, Kim Y, Kim M, Oh EJ, et al. (2014) New proposed guidelines for early identification of successful myeloid and erythroid engraftment in hematopoietic stem cell transplantation. J Clin Lab Anal 28: 469-477.

19. Ahmed RAR, Fayek MHA, Salem DAD (2020) Using reticulocyte hemoglobin equivalent (RET- $\mathrm{He})$ in diagnosis of iron deficiency anemia. QJM: An International Journal of Medicine.

20. Chinudomwong P, Binyasing A, Trongsakul R, Paisooksantivatana K (2020) Diagnostic performance of reticulocyte hemoglobin equivalent in assessing the iron status. Journal of Clinical Laboratory Analysis 34: e23225.
21. van der Linden N, Klinkenberg LJJ, Meex SJR, Beckers EAM, de Wit NCJ, et al. (2014) Immature platelet fraction measured on the Sysmex XN hemocytometer predicts thrombopoietic recovery after autologous stem cell transplantation. Eur J Haematol 93: 150-156.

22. Takami A, Shibayama M, Orito M, Omote M, Okumura H, et al. (2007) Immature platelet fraction for prediction of platelet engraftment after allogeneic stem cell transplantation. Bone Marrow Transplant 39: 501-507.

23. Zucker ML, Murphy CA, Rachel JM, Martinez GA, Abhyankar S, et al. (2006) Immature platelet fraction as a predictor of platelet recovery following hematopoietic progenitor cell transplantation. Lab Hematol 12: 125-130.

24. Buttarello M, Mezzapelle G, Freguglia F, Plebani M (2020) Reticulated platelets and immature platelet fraction: Clinical applications and method limitations. Int $\mathrm{J}$ Lab Hematol 42: 363-370.

25. Ko YJ, Kim H, Hur M, Choi SS, Moon HW, et al. (2013) Establishment of reference interval for immature platelet fraction. Int J Lab Hematol 35: 528-533. 